\title{
Energy Momentum Tensor Generates Repulsive Gravitational Force
}

\author{
Branko M. Novakovic
}

\begin{abstract}
Solution of the field equations by including gravitational energy - momentum tensor in it (but without cosmological constant $\Lambda$ ) generates repulsive gravitational force. This repulsive force could be the source of the dark energy. Regularity condition of the related line element gives the limitations to the gravitational radius $G M / 2 c^{2} \leq r<\infty$. This means that presented solution has no singularity points. Minimal diameter of the universe mass $2 r=G M / c^{2}$ corresponds to the Planck's length $\mathrm{L}_{\mathrm{pl}}=\mathrm{GM}_{\mathrm{pl}} / \mathrm{c}^{2}$, as the minimal diameter of the Planck's mass $M_{\mathrm{pl}}$. From the universe velocity equation, we obtain the limitations to the energy conservation constant $0<\kappa$ $\leq 1$. This means that the kinetic energy is less or equal to the potential energy. For that case the spatial curvature of the space is $\mathcal{K} \geq 0$. In other words, our universe is a flat or a hyperspherical, because hyperbolic universe is excluded. Further, the zero points of the universe velocity equation determine the minimal gravitational radius at $\mathbf{r}_{\min }=$ $\mathbf{G M} /(1+\kappa) \mathbf{c}^{2}$ and maximal gravitational radius at $r_{\max }=$ $G M /(1-\kappa) c^{2}$. Applying radial density $\rho_{r}=M / r$ to the minimal and maximal gravitational radiuses we obtain the other limitations to the energy conservation constant $0<\kappa<1$ and to the spatial curvature of the space $\mathcal{K}>0$. In that case our universe is a hyperspherical because flat and hyperbolic universes are excluded by the mentioned limitations.
\end{abstract}

Index Terms- Universe expansion; Repulsive gravitational force; Dark energy; Minimal gravitational radius; Maximal gravitational radius

\section{INTRODUCTION}

As it is well known today, the universe continues to expand, even at an accelerating rate. The main reason for it is an unknown energy called a dark energy. This energy occupies about 68 percent of the total energy in the universe. There exist more dynamic models of the universe motion. The most known is the model that has been developed independently by Alexander Friedmann [1,2], Georges Lemaitre [3,4], Howard Percy Robertson [5-7] and Arthur Geoffrey Walker [8]. Therefore, it has been named Friedmann - Lemaitre Robertson - Walker (FLRW) model. In the literature one can find also the names Friedmann - Robertson - Walker (FRW), or Robertson - Walker (RW), or Friedmann - Lemaitre (FL) model. In the modern cosmology it is also called Standard Model (ST) [9]. The FLRW model describes a homogeneous, isotropic expanding or contracting universe. The general metric has been introduced on the assumption that the universe geometric properties are homogeneous and isotropic, i.e. the Cosmological Principle is valued. Empirically, this is justified on scales larger than $\sim 100 \mathrm{Mpc}$. They also used the Einstein field equations [10,11] for derivation of the "scale factor" of the universe as a function of

Branko Novakovic, FSB - University of Zagreb, Luciceva 5, P.O.B. 509, 10000 Zagreb, Croatia. time. The FLRW model is well described in the references $[1-9,12-18]$.

The history and present state of the universe motion models have been presented in [1-41]. The main problem of the all models of the universe motion is to answer (among the others) to the important questions of the cosmology [23]: what occurred at the initial singularity?, how old is the universe?, how big is the universe? and what is its ultimate fate? Recently, a new method for testing of the Cosmological Principle by using an isotropic blackbody cosmic microwave background radiation, as evidence for a homogeneous universe, has been presented in [33]. Today we know that the universe continues to expand, even at an accelerating rate. This acceleration is caused by an unknown energy called a dark energy. But we do not know the source of dark energy. One of the candidates for the source of dark energy is a cosmological constant $\Lambda$.

Here we derived a new model of the universe motion based on the solution of the field equations by including gravitational energy - momentum tensor in it, but without cosmological constant $\Lambda$. This solution showed that the gravitational energy - momentum tensor generates repulsive gravitational force. This force is repulsive in the region $G M / 2 c^{2} \leq r<G M / c^{2}$. At the radius $r=G M / 2 c^{2}$ repulsive gravitational force is maximal and at the radius $r=G M / c^{2}$ is equal to zero. Further, in the region $G M / c^{2}<r<\infty$ the gravitational force is attractive as we know from the experience. At the radius $r=G M / c^{2}$ gravitational force is changing from repulsive to the attractive one (in the expanding phase) and from attractive to repulsive force (in the contracting phase). Following the observations, we know that nowadays our universe is expanding in the acceleration rate. Thus, we can conclude that the present universe radius should be less than $G M / c^{2}$. From the previous consideration we can see that the repulsive gravitational energy has the role of dark energy. In that sense, one can conclude that the repulsive gravitational force could be the source of the dark energy. Thus, the gravitational energy - momentum tensor has an effect as feedback in order to control of the universe motion.

Further, the regularity condition of the related line element gives the limitations to the energy conservation constant $0<\kappa$ $\leq 1$. This means that the kinetic energy is less or equal to the potential energy. For that case the spatial curvature of the space $\mathcal{K} \geq 0$. In other words, our universe is a flat or a hyperspherical, because hyperbolic universe is excluded. The zero points of the universe velocity equation determine the minimal gravitational radius at $r_{\min }=G M /(1+\kappa) c^{2}$ and maximal gravitational radius at $r_{\max }=G M /(1-\kappa) c^{2}$. Applying radial density $\rho_{r}=M / r$ to the minimal and maximal gravitational radiuses we obtain the other limitations to the 
energy conservation constant $0<\kappa<1$ and to the spatial curvature of the space $\mathcal{K}>0$. In this case our universe is a hyperspherical because flat and hyperbolic universes are excluded by the limitations of the energy conservation constant.

The important consequence of the solution of the field equations by including gravitational energy - momentum tensor in it is the existence of the minimal gravitational radius at $r=G M / 2 c^{2}$. This is very important information, because it tells us that the gravitational field has no singularity point. Meanwhile, the presented dynamic model of the universe motion describes the motion after universe creation by Big Bang, from the moment when gravitational repulsive force became dominant in a space - time.

For derivation of the equations of the universe motion we used the related Lagrangean based on the solution of the line element in a gravitational field. In order to compare this solution of the universe motion with one of the existing solutions, here we started with the Friedmman's model of the universe motion.

\section{FRIEDMAN EQUATIONS}

Following the assumption that the universe geometric properties are homogeneous and isotropic, i.e. the Cosmological Principle is valid, Friedman has been derived his equations $[1,2]$ :

$$
\begin{aligned}
& \left(\frac{\dot{\alpha}}{\alpha}\right)^{2}+\frac{\mathcal{K} c^{2}}{\alpha^{2}}-\frac{\Lambda c^{2}}{3}=\frac{8 \pi G}{3} \rho, \\
& \frac{\ddot{\alpha}}{\alpha}=-\frac{4 \pi G}{3}\left(\rho+\frac{3 p}{c^{2}}\right)+\frac{\Lambda c^{2}}{3} .
\end{aligned}
$$

Here $\alpha(t)$ is the scale factor with related time derivations $\dot{\alpha}$ and $\ddot{\alpha}, \mathcal{K}$ is spatial curvature parameter, $\Lambda$ is cosmological constant, while $\rho$ and $p$ are fluid mass density and pressure, respectively. The presented equations (1) are the basis of the standard big bang cosmological model including the current $\Lambda \mathrm{CDM}$ model. Following the mentioned assumption that the universe is isotropic and homogenous, the model (1) can be used as a first approximation for the evolution of the real, lumpy universe, because it is simple for calculation. Further more, the models which calculate the lumpiness in the universe can be added to this model as extensions.

The second equation in (1) states that both the energy density and the pressure cause the expansion rate of the universe, $\ddot{\alpha}$, to decrease. It means that both the energy density and the pressure cause a deceleration in the expansion of the universe. This is the consequence of gravitation, including that pressure is playing a similar role to that of energy (or mass) density. This is, of course, in accordance with the principles of general relativity. On the other side, the cosmological constant, $\Lambda$, causes acceleration in the expansion of the universe.

Friedmann also used the full form of the Einstein's field equations in the General Theory of Relativity that is given by the relation $[10,11]$

$$
R_{\mu \eta}-\frac{1}{2} g_{\mu \eta} R+\Lambda g_{\mu \eta}=\frac{8 \pi G}{c^{4}} T_{\mu \eta}, \mu, \eta=0,1,2,3 .
$$

In this relation $R_{\mu \eta}$ is Ricci tensor, $g_{\mu \eta}$ is metric tensor, $R$ is Ricci scalar, $\Lambda$ is the Einstein's cosmological constant, $G$ is the Newton's gravitational constant, $c$ is the speed of the light in a vacuum and $T_{\mu \eta}$ is the energy-momentum tensor. Determination of the time evolution of the scale factor $\alpha(t)$ requires the Einstein's field equations together with a way of calculation of density, $\rho(t)$, such as a cosmological equation of state. If the energy-momentum tensor, $T_{\mu \eta}$, is similarly assumed to be isotropic and homogenous, then we have an analytic solution to Einstein's field equations (2), giving the Friedman equations in the form (1).

\section{UNIVERSE MOTION EQUATIONS BASED ON GRAVITATIONAL ENERGY MOMENTUM TENSOR}

Here we show new approach to the description of the universe motion. This approach is based on the solution of the field equations (2) with inclusion of the gravitational energy momentum tensor $T_{\mu \eta}$, but without cosmological constant $\Lambda$. On that way we obtain the solution of the field parameters in a gravitational field. Further, for derivation of the equations of the universe motion we used the related LaGrangean based on the solution of the line element in a gravitational field.

The general nondiagonal form of the line element, $\mathrm{ds}^{2}$, in the spherical polar coordinates, can be described by the equation

$$
d s^{2}=-v c^{2} d t^{2}+2 \lambda c d t d r+d r^{2}+r^{2} d \theta^{2}+r^{2} \sin ^{2} \theta d \phi^{2} .
$$

Here $v$ and $\lambda$ are field parameters, $c$ is the speed of the light in a vacuum, $r$ is a radius vector, $\theta$ is an angle between radius vector $r$ and $z$-axis, and $\phi$ is an angle between projection of a radius vector $r$ on $(x-y)$ plane and $x$-axis. Applying field equations

$$
R_{\mu \eta}-\frac{1}{2} g_{\mu \eta} R=\frac{8 \pi G}{c^{4}} T_{\mu \eta}, \quad \mu, \eta=0,1,2,3 .
$$

we obtain the solutions of the field parameters $v$ and $\lambda$ :

$$
\begin{gathered}
T_{\mu \eta} \neq 0, \quad \Lambda=0, \quad \rightarrow \quad \nu=1-\frac{2 G M}{r c^{2}}+\left(\frac{G M}{r c^{2}}\right)^{2}, \\
\lambda=\sqrt{\frac{2 G M}{r c^{2}}-\left(\frac{G M}{r c^{2}}\right)^{2}} .
\end{gathered}
$$

Here $G M / c^{2}$ is the Newton's constant of integration, $G$ is a gravitational constant, $M$ is a total gravitational mass, $r$ is a gravitational radius and $c$ is the speed of the light in a vacuum. If displacement four-vector $d X$ is defined in frame $K$ by the expression

$$
d X \rightarrow K(c d t, d r, d \theta, d \phi)=\left\{d x^{i}\right\}, \quad i=0,1,2,3,
$$

then the related covariant metric tensor of the line element (3) has the following matrix presentation 


$$
\left[g_{\mu \eta}\right]=\left[\begin{array}{cccc}
-v & \lambda & 0 & 0 \\
\lambda & 1 & 0 & 0 \\
0 & 0 & r^{2} & 0 \\
0 & 0 & 0 & r^{2} \sin ^{2} \theta
\end{array}\right] .
$$

Here the non-null components of the metric tensor $g_{\mu \eta}$ are given by the relations:

$$
\begin{gathered}
g_{00}=-v=-\left(1-\frac{2 G M}{r c^{2}}+\left(\frac{G M}{r c^{2}}\right)^{2}\right), g_{11}=1, \\
g_{22}=r^{2}, g_{01,10}=\lambda=\sqrt{\frac{2 G M}{r c^{2}}-\left(\frac{G M}{r c^{2}}\right)^{2}}, \\
g_{33}=r^{2} \sin ^{2} \theta .
\end{gathered}
$$

The related determinant of the metric tensor (7) has the form:

$$
\begin{gathered}
\operatorname{det}\left[g_{\mu \eta}\right]=-r^{4}\left(v+\lambda^{2}\right) \sin ^{2} \theta, \quad r=1, \theta=\frac{\pi}{2}, \rightarrow \\
\operatorname{det}\left[g_{\mu \eta}\right]=-\left(v+\lambda^{2}\right)=-1, \quad v=1-\lambda^{2} .
\end{gathered}
$$

In the previous relation we use the normalization for $r=1$, and $\theta=\pi / 2$. On that way, the well-known condition for the metric tensor of the line element, det $\left(g_{\mu \eta}\right)=-1$, is satisfied. As the result we obtain the simple relation between field parameters $v$ and $\lambda$.

By including metric parameters (8) and (9) into the field equations (4), we obtain the related gravitational energy-momentum tensor (EMT)

$$
\left[T_{\mu \eta}\right]=\left[\begin{array}{rrcc}
\nu & -\lambda & 0 & 0 \\
-\lambda & -1 & 0 & 0 \\
0 & 0 & r^{2} & 0 \\
0 & 0 & 0 & r^{2} \sin ^{2} \theta
\end{array}\right] \frac{(G M)^{2}}{8 \pi G r^{4}}
$$

It is easy to prove that this EMT satisfies the required properties: 1) symmetry, $T_{\mu \eta}=T_{\eta \mu} ; 2$ ) non-negative energy density for static and free field, $T_{00} \geq 0$; and 3 ) zero trace, $T=0$.

Proposition 1. If the line element is defined by the relations (3) to (9), then the dynamic model of the universe motion, for $T_{\mu \eta} \neq 0$ and $\Lambda=0$, is given by the equations:

$$
\begin{aligned}
& \left(\frac{\dot{r}}{r}\right)^{2}+\frac{\mathcal{K} c^{2}}{r^{2}}=\frac{8 \pi G}{3} \rho\left(1-\frac{2 \pi G}{3} \frac{\rho r^{2}}{c^{2}}\right), \\
& \frac{\ddot{r}}{r}=-\frac{4 \pi G}{3}\left(\rho+\frac{3 p}{c^{2}}\right)\left(1-\frac{4 \pi G}{3} \frac{\rho r^{2}}{c^{2}}\right) .
\end{aligned}
$$

Comparing the first equation in (11) with the first Friedman equation in (1), we can conclude that both equations have the same form of the two parts of the left side and the first part of the right side if the substitutions $r=\alpha$ and $\dot{r}=\dot{\alpha}$ are valid. Comparing the second equation in (11) with the second Friedman equation in (1), we can conclude that both equations have the same form of the left side and of the first part of the right side if the substitutions $r=\alpha$ and $\ddot{r}=\ddot{\alpha}$ are valid. Thus, if the equations (11) describe the universe motion, then the radial coordinate $r(\mathrm{t})$ has the roll of the scale factor $\alpha(t)$. The first part on the right side of the second equation in (11) states that both the energy density and the pressure cause a deceleration in the expansion of the universe. This is the consequence of gravitation, including that pressure is playing a similar role to that of energy (or mass) density. This is, of course, in accordance with the principles of general relativity. On the other hand, the second part of the right side of the second equation in (11) causes acceleration in the expansion of the universe. This is the consequence of the inclusion of the gravitational energy momentum tensor on the right side of the field equations that generates the repulsive gravitation force in a gravitational field. This fact tells us that the repulsive gravitational force could be the source of the dark energy. Of course, this should be confirmed by the related experiments.

Proof of the Proposition 1. In order to prove of the proposition 1 , for the case $\left(T_{\mu \eta} \neq 0\right.$ and $\left.\Lambda=0\right)$, one can start with the Lagrangean of the line element (3)

$$
\begin{gathered}
L=\left[-\frac{d s^{2}}{c^{2} d \tau^{2}}\right]^{1 / 2}=\frac{1}{c}\left[-g_{\mu \eta} \frac{d x^{\mu}}{d \tau} \frac{d x^{\eta}}{d \tau}\right]^{1 / 2}, \\
\mu, \eta=0,1,2,3 .
\end{gathered}
$$

Here $d \tau$ is the differential of the proper time $\tau$ and $d x^{\mu}$, and $d x^{\eta}$, are components of the contravariant displacement four-vector $d X$ in (6). Applying the field parameters (8) and using (12) one obtains the Lagrangean in the following form

$$
L=\left(\begin{array}{c}
\left.v\left(\frac{d t}{d \tau}\right)^{2}-\frac{1}{c^{2}} 2 \lambda c\left(\frac{d t}{d \tau}\right)\left(\frac{d r}{d \tau}\right)-\frac{1}{c^{2}}\left(\frac{d r}{d \tau}\right)^{2}-\right)^{1 / 2} . \\
-\frac{r^{2}}{c^{2}}\left(\frac{d \theta}{d \tau}\right)^{2}-\frac{r^{2}}{c^{2}} \sin ^{2} \theta\left(\frac{d \phi}{d \tau}\right)^{2}
\end{array}\right)^{2}
$$

The related Euler - Lagrange equations are given by the expressions:

$$
\begin{gathered}
\frac{\partial L}{\partial z^{i}}=\frac{d}{d \tau}\left(\frac{\partial L}{\partial \dot{z}^{i}}\right), \quad i=0,1,2,3, \quad z^{o}=t, \\
z^{1}=r, \quad z^{2}=\theta, \quad z^{3}=\phi, \quad \dot{z}^{i}=\frac{d z^{i}}{d \tau} .
\end{gathered}
$$

Applying $i=0$ to the relation (14) one obtains energy conservation equation:

$$
\begin{aligned}
\frac{\partial L}{\partial t}=0 \rightarrow \frac{\partial L}{\partial \dot{t}}=\text { const. } & =\kappa \rightarrow v \frac{d t}{d \tau}-\frac{\lambda}{c} \frac{d r}{d \tau}=\kappa, \\
\frac{d \kappa}{d \tau} & =\dot{\kappa}=0 .
\end{aligned}
$$

Here parameter $\kappa$ is the energy conservation constant. Applying $i=3$ to the relation (14) one obtains angular momentum conservation equation: 


$$
\begin{aligned}
& \frac{\partial L}{\partial \phi}=0 \rightarrow \frac{\partial L}{\partial \dot{\phi}}=\text { const. }=h \rightarrow \\
& -\frac{r^{2} \dot{\phi}^{2} \sin ^{2} \theta}{c^{2}}=h, \quad \frac{d h}{d \tau}=\dot{h}=0 .
\end{aligned}
$$

Parameter $h$ is the angular momentum conservation constant. In the case $\theta=\pi / 2$ (as in Newtonian theory) the angular momentum conservation equation (16) is transformed into the well-known relation:

$$
\begin{gathered}
\frac{\partial L}{\partial \phi}=0 \rightarrow \frac{\partial L}{\partial \dot{\phi}}=\text { const. }=h \rightarrow \\
-\frac{r^{2} \dot{\phi}^{2}}{c^{2}}=h, \quad \frac{d h}{d \tau}=\dot{h}=0 .
\end{gathered}
$$

Now, substituting the relations derived from (15) and (9), respectively:

$$
\frac{d t}{d \tau}=\left(\kappa+\frac{\lambda}{c} \frac{d r}{d \tau}\right) v^{-1}, \quad v=1-\lambda^{2},
$$

into the equation (13), and employing $\varepsilon=L$ (where $\varepsilon=1$ for a time-like geodesics and $\varepsilon=0$ for a null geodesics) one obtains the following relation

$$
\begin{gathered}
\frac{1}{2}\left[\dot{r}^{2}+r^{2}\left(\dot{\theta}^{2}+\dot{\phi}^{2} \sin ^{2} \theta\right)\left(1-\lambda^{2}\right)\right] \\
-\frac{\lambda^{2} \varepsilon^{2} c^{2}}{2}=\frac{c^{2}}{2}\left(\kappa^{2}-\varepsilon^{2}\right), \\
\dot{r}=\frac{d r}{d \tau}, \quad \dot{\theta}=\frac{d \theta}{d \tau}, \quad \dot{\phi}=\frac{d \phi}{d \tau} .
\end{gathered}
$$

This relation represents the generalized energy equation of a particle with unit mass, where the sum of the kinetic energy $E_{k}$ and potential energy $E_{p}$ is equal to constant:

$$
\begin{gathered}
E_{k}=\frac{1}{2}\left[\dot{r}^{2}+r^{2}\left(\dot{\theta}^{2}+\dot{\phi}^{2} \sin ^{2} \theta\right)\left(1-\lambda^{2}\right)\right], \\
E_{p}=-\frac{\lambda^{2} \varepsilon^{2} c^{2}}{2}, \frac{c^{2}}{2}\left(\kappa^{2}-\varepsilon^{2}\right)=\text { const. }, \\
\rightarrow E_{k}+E_{p}=\text { const } .
\end{gathered}
$$

For the case $\left(T_{\mu \eta} \neq 0\right.$ and $\left.\Lambda=0\right)$, the relation (19) is transformed into the new form of the generalized energy equation

$$
\begin{gathered}
\frac{1}{2}\left[\dot{r}^{2}+r^{2}\left(\dot{\theta}^{2}+\dot{\phi}^{2} \sin ^{2} \theta\right)\left(1-\frac{2 G M}{r c^{2}}+\left(\frac{G M}{r c^{2}}\right)^{2}\right)\right] \\
-\frac{1}{2}\left(\frac{2 G M}{r c^{2}}-\left(\frac{G M}{r c^{2}}\right)^{2}\right) \varepsilon^{2} c^{2}=\frac{c^{2}}{2}\left(\kappa^{2}-\varepsilon^{2}\right) .
\end{gathered}
$$

This relation also represents that the sum of the kinetic energy $E_{k}$ and potential energy $E_{p}$ of a particle with unit mass is equal to constant

$$
\begin{aligned}
& E_{k}=\frac{1}{2}\left[\dot{r}^{2}+r^{2}\left(\dot{\theta}^{2}+\dot{\phi}^{2} \sin ^{2} \theta\right)\left(1-\frac{2 G M}{r c^{2}}+\left(\frac{G M}{r c^{2}}\right)^{2}\right)\right] \\
& E_{p}=-\frac{1}{2}\left(\frac{2 G M}{r c^{2}}-\left(\frac{G M}{r c^{2}}\right)^{2}\right) \varepsilon^{2} c^{2}, \frac{c^{2}}{2}\left(\kappa^{2}-\varepsilon^{2}\right)=\text { const. }
\end{aligned}
$$

Now, one can assume that the motion is in the radial direction, only. This means that $\dot{\theta}=0$ and $\dot{\phi}=0$. For that case, the generalized energy equation (19) is transformed into the relation

$$
\frac{\dot{r}^{2}}{2}-\frac{\lambda^{2} \varepsilon^{2} c^{2}}{2}=\frac{c^{2}}{2}\left(\kappa^{2}-\varepsilon^{2}\right) .
$$

In the case $\left(T_{\mu \eta} \neq 0\right.$ and $\left.\Lambda=0\right)$, the relation (23) is given by the equation

$$
\frac{\dot{r}^{2}}{2}-\frac{1}{2}\left(\frac{2 G M}{r c^{2}}-\left(\frac{G M}{r c^{2}}\right)^{2}\right) \varepsilon^{2} c^{2}=\frac{c^{2}}{2}\left(\kappa^{2}-\varepsilon^{2}\right) .
$$

Now one can apply a mass density $\rho$, spatial curvature constant $\mathcal{K}$, and $\varepsilon=1$ (for time-like geodesics):

$$
\begin{gathered}
\frac{2 G M r^{2}}{r^{3} c^{2}}=\frac{8 \pi G}{3 c^{2}} \rho r^{2}, \quad \frac{G M r^{2}}{r^{3} c^{2}}=\frac{4 \pi G}{3 c^{2}} \rho r^{2}, \\
\left(\frac{G M r^{2}}{r^{3} c^{2}}\right)^{2}=\left(\frac{4 \pi G}{3 c^{2}} \rho r^{2}\right)^{2},\left(\varepsilon^{2}-\kappa^{2}\right)=\left(1-\kappa^{2}\right)=\mathcal{K} .
\end{gathered}
$$

By including the substitutions from (25), the equation (24) is transformed into the relation

$$
\left(\frac{\dot{r}}{r}\right)^{2}+\frac{\mathcal{K} c^{2}}{r^{2}}=\frac{8 \pi G}{3} \rho\left(1-\frac{2 \pi G}{3} \frac{\rho r^{2}}{c^{2}}\right) .
$$

Comparing this equation with the first Friedman equation in (1), we can conclude that both equations have the same form of the two parts of the left side and the first part of the right side if the substitutions $r=\alpha$ and $\dot{r}=\dot{\alpha}$ are valid. Thus, if the equation (26) describes the universe motion, then the radial coordinate $r(t)$ has the roll of the scale factor $\alpha(t)$.

Applying a time derivative to (26) one obtains the following acceleration equation

$$
\frac{\ddot{r}}{r}=\frac{4 \pi G}{3}\left(\dot{\rho} \frac{r}{\dot{r}}+2 \rho\right)\left(1-\frac{4 \pi G}{3} \frac{\rho r^{2}}{c^{2}}\right) .
$$

In order to calculate a time derivative of the mass density $\dot{\rho}$, one should assume that the expansion of the universe is an adiabatic process. In that case, the thermo-dynamical approach can be considered. This is equivalent to the first law of thermodynamics and is resulting with the relations:

$$
\begin{gathered}
\dot{\rho}=-3 \frac{\dot{\alpha}}{\alpha}\left(\rho+\frac{p}{c^{2}}\right), \alpha \rightarrow r, \dot{\alpha} \rightarrow \dot{r}, \rightarrow \\
\dot{\rho}=-3 \frac{\dot{r}}{r}\left(\rho+\frac{p}{c^{2}}\right) .
\end{gathered}
$$


Including the second equation from (28) into (27) one obtains the following relation

$$
\frac{\ddot{r}}{\mathrm{r}}=-\frac{4 \pi G}{3}\left(\rho+\frac{3 p}{c^{2}}\right)\left(1-\frac{4 \pi G}{3} \frac{\rho r^{2}}{c^{2}}\right) .
$$

Comparing this equation with the second Friedman equation in (1), we can conclude that both equations have the same form of the left side and on the first part of the right side if the substitutions $r=\alpha$ and $\ddot{r}=\ddot{\alpha}$ are valid. Thus, if the equation (29) describes the universe motion, then the radial coordinate $r(\mathrm{t})$ has the roll of the scale factor $\alpha(\mathrm{t})$. The equation (29) states that the first part of the right side causes a deceleration in the expansion of the universe. On the other hand, the second part of the right-side gives the conditions for the universe expansion, contraction and null acceleration:

$$
\begin{gathered}
\rho>\frac{3 c^{2}}{4 \pi G r^{2}} \rightarrow \text { expansion, } \\
\rho<\frac{3 c^{2}}{4 \pi G r^{2}} \rightarrow \text { contraction, } \\
\rho=\frac{3 c^{2}}{4 \pi G r^{2}} \rightarrow \text { null acceleration } .
\end{gathered}
$$

At the point of null acceleration, we have change from the expansion to the contraction and vice versa.

From the previous consideration one can take the equations (26) and (29) and put them together:

$$
\begin{aligned}
& \left(\frac{\dot{r}}{r}\right)^{2}+\frac{\mathcal{K} c^{2}}{r^{2}}=\frac{8 \pi G}{3} \rho\left(1-\frac{2 \pi G}{3} \frac{\rho r^{2}}{c^{2}}\right), \\
& \frac{\ddot{r}}{r}=-\frac{4 \pi G}{3}\left(\rho+\frac{3 p}{c^{2}}\right)\left(1-\frac{4 \pi G}{3} \frac{\rho r^{2}}{c^{2}}\right) .
\end{aligned}
$$

The first relation in (30) is the velocity equation, while the second one is the acceleration equation. The both equations are valid for the case $\left(T_{\mu \eta} \neq 0\right.$ and $\left.\Lambda=0\right)$. Thus, the Proposition 1 , related to the case where repulsive gravitational force plays the role of the dark energy, is finished by the relations (30). This fact tells us that the repulsive gravitational force could be the source of the dark energy. Of course, this should be confirmed by the related experiments.

\section{CONSEQUENCES OF THE UNIVERSE MOTION MODEL}

\section{A. Regularity of the metrics of the line element}

The line element (3) with parameters (5) can be rewritten into the following form

$$
\begin{aligned}
& d s^{2}=-\left(1-\frac{G M}{r c^{2}}\right)^{2} c^{2} d t^{2}+2 \sqrt{\frac{2 G M}{r c^{2}}\left(1-\frac{G M}{2 r c^{2}}\right)} c d t d r \\
& +d r^{2}+r^{2} d \theta^{2}+r^{2} \sin ^{2} \theta d \phi^{2} .
\end{aligned}
$$

It is easy to prove that the line element (31) is regular if the following conditions are satisfied:

$$
\left(1-\frac{G M}{2 r c^{2}}\right) \geq 0 \rightarrow \frac{G M}{2 r c^{2}} \leq 1 \rightarrow \frac{G M}{2 c^{2}} \leq r<\infty .
$$

Thus, the region of the regularity of the line element (31) is determined by the last relation in (32). Further, from the previous relations we can conclude that there exists a minimal gravitational radius $r_{\text {min }}$ which still preserves regularity of the line element (31)

$$
r_{\text {min }}=\frac{G M}{2 c^{2}} \text {. }
$$

Including of the minimal gravitational radius (33) into the line element (31) we obtain regular line element in the form

$$
d s^{2}=-c^{2} d t^{2}+d r^{2}+r^{2} d \theta^{2}+r^{2} \sin ^{2} \theta d \phi^{2} .
$$

For the radiuses less than minimal gravitational radius $r_{\text {min }}$ the line element (31) becomes imaginary item. Gravitational radius can be equal to zero only if the mass is equal to zero. This means that any mass $m \neq 0$ can not reach the singularity at $r=0$ in a gravitational field. In that sense, dynamic model of the universe motion $(11,30)$ describes the motion after universe creation by Big Bang, at the moment when gravitational repulsive force became dominant in a space - time. The minimal gravitational radius is four time less than Schwarzschild radius

$$
r_{s}=\frac{2 G M}{c^{2}}=4 r_{\min }=4 \frac{G M}{2 c^{2}}
$$

\section{B. The physical meaning of the minimal gravitational radius}

In order to highlight the physical meaning of the minimal gravitational radius $r_{\min }$, one can apply of the Planck's mass $M_{p}$ to the relation (33)

$$
r_{p \min }=\frac{G M_{p}}{2 c^{2}} .
$$

Here $r_{p \min }$ is a minimal radius of the Planck' mass $M_{p}$. As it is well-known, the Planck's length $L_{p}$ [42-44] and the Planck's mass $M_{p}$ [45] are defined from three fundamental physical constants: the speed of light in vacuum $c$, the reduced Planck's constant $\hbar$ and the gravitational constant $G$ :

$$
M_{p}=\sqrt{\frac{\hbar c}{G}}, \quad L_{p}=\sqrt{\frac{\hbar G}{c^{3}}} .
$$

Assuming that the Planck's mass $M_{p}$ is spherically symmetric body (i.e. $L_{p}$ is diameter of the Planck's mass) and following the relations (36) and (37), we can calculate the following equality:

$$
\begin{gathered}
\frac{M_{p}}{2 r_{p \min }}=\frac{c^{2}}{G}, \quad \frac{M_{p}}{L_{p}}=\frac{\sqrt{\hbar c / G}}{\sqrt{\hbar G / c^{3}}}=\frac{c^{2}}{G}=\text { const } . \\
\rightarrow 2 r_{p \min }=L_{p} \rightarrow r_{p \min }=\frac{L_{p}}{2} .
\end{gathered}
$$

From the last relation in (38), one can conclude that the minimal gravitational radius of the Planck's mass $M_{p}$, denoted by $r_{p \min }$, is equal to the half of the Planck's length $L_{p}$. 
Thus, the physical meaning of the Planck's length could be: $L_{p}$ is the gravitational minimal diameter (length) of the Planck's mass $M_{p}$. On the other words, Planck's Mass $M_{p}$ can not have a gravitational diameter less than Planck's length $L_{p}$. Furthermore, from the relation (33) we can see that the minimal radius is proportional to the gravitational mass. Thus, the smallest minimal radius belongs to the smallest mass in space - time. Since the Planck's mass is not the smallest mass in space - time, the Planks length is not the smallest length in that sense.

From the relation (33) one can derive maximal radial density in gravitational field $\rho_{r_{\max }}$ :

$$
\begin{gathered}
\frac{M}{2 r_{\text {min }}}=\frac{M}{L_{\text {min }}}=\frac{c^{2}}{G}=\text { const. } \rightarrow \\
\rho_{r_{\text {max }}}=\frac{M}{r_{\text {min }}}=\frac{M_{p}}{r_{p \min }}=\frac{2 c^{2}}{G}=\text { const } .
\end{gathered}
$$

Thus, the minimal gravitational radius of mass $M$ corresponds to the minimal gravitational radius $r_{p m i n}$ of the Planck's mass $M_{p}$. On the same way, the minimal gravitational length $L_{\min }$ is the minimal diameter of the mass $M$. From relations (39) we can see that the maximal radial density $\rho_{\text {rmax }}$ is at the minimal gravitational radius and is the constant and the same for all masses including Planck's mass.

\section{Universe velocity and acceleration at the characteristic radiuses}

The relation (24) represents that the sum of the kinetic energy $E_{k}$ and potential energy $E_{p}$ of a particle with unit mass is equal to constant:

$$
\begin{gathered}
E_{k}=\frac{1}{2}\left[\dot{r}^{2}\right], \quad E_{p}=-\frac{1}{2}\left(\frac{2 G M}{r c^{2}}-\left(\frac{G M}{r c^{2}}\right)^{2}\right) \varepsilon^{2} c^{2}, \\
E_{k}+E_{p}=\frac{c^{2}}{2}\left(\kappa^{2}-\varepsilon^{2}\right)=\text { const. }
\end{gathered}
$$

Following (40) and putting that $\varepsilon=1$ (for time-like geodesics), one obtains the following relations between kinetic and potential energies and the energy conservation constant $\kappa$ :

$$
\begin{gathered}
E_{k}+E_{p}=\frac{c^{2}}{2}\left(\kappa^{2}-1\right),\left|E_{k}\right|=\left|E_{p}\right| \rightarrow \kappa=1, \\
\left|E_{k}\right|>\left|E_{p}\right| \rightarrow \kappa>1,\left|E_{k}\right|<\left|E_{p}\right| \rightarrow 0<\kappa<1 .
\end{gathered}
$$

Following the relations (24) and (40) and assuming that the universe motion follows time-like geodesic $(\varepsilon=1)$, one can derive radial velocity $\dot{r}$ and radial acceleration $\ddot{r}$ of the universe motion:

$$
\begin{gathered}
\dot{r}= \pm\left[\frac{2 G M}{r}\left(1-\frac{G M}{2 r c^{2}}\right)+\left(\kappa^{2}-1\right) c^{2}\right]^{1 / 2}, \\
\ddot{r}=-\frac{G M}{r^{2}}\left(1-\frac{G M}{r c^{2}}\right) .
\end{gathered}
$$

The sign (+) in the velocity equation is valid for an expanding phase, while the sign (-) is related to the contracting one. The acceleration equation tells us that the universe acceleration becomes repulsive if $\left(G M / r c^{2}\right)>1$. One of the conclusions could be that the repulsive gravitational force is the source of dark energy. For the case $\left(G M / r c^{2}\right)=1$ the acceleration is equal to zero and for $\left(G M / r c^{2}\right)<1$ the acceleration is attractive. At the point $\left(G M / r c^{2}\right)=1$, the repulsive acceleration is changing into the attractive one (for expansion phase) and vice versa (for contraction phase).

The velocity equation in (42) has two zeros at the positions $r_{1}$ and $r_{2}$ :

$$
r_{1}=\frac{G M}{(1+\kappa) c^{2}}, \quad r_{2}=\frac{G M}{(1-\kappa) c^{2}}
$$

From the relations (24) and (43) one can see that the hyperspherical scenario of the universe motion can be realized only for the case where the energy conservation constant $\kappa$ is satisfying the condition $(0<\kappa<1)$. This means that potential energy is greater than kinetic energy. For that case the velocity equation in (42) has two finite zeros: initial one for $r=r_{1}$, and final one for $r=r_{2}$.

On the other side the hyperbolic scenario of the universe motion can be realized only for the case where the energy conservation constant $\kappa$ is greater than one $(\kappa>1)$. This means that kinetic energy is greater than potential energy. For that case the velocity relation (42) has only one real zero $r_{1}$ in (43), because the other one gives a negative $r_{2}$. The real zero $r_{1}$ is the initial one for this scenario of the universe motion.

Finally, the flat universe can be realized only for the case where the energy conservation constant $\kappa$ is equal to one ( $\kappa$ $=1$ ). This means that kinetic energy is equal to potential energy. For that case the velocity equation in (42) has one finite initial zero for $r=r_{1}$, and one infinite final zero for $r_{2} \rightarrow$ $\infty$. The initial radius $r_{l}$ of the hyperbolic universe $(\kappa>1)$ is less than the initial radius of the flat universe $(\kappa=1)$ and also less than initial radius of the hyperspherical universe $(\kappa<1)$.

The maximal radial velocity $\dot{r}_{\max }$ can be obtained from (42) by applying condition that the radial universe acceleration is equal to zero:

$$
\ddot{r}=0 \rightarrow r=r_{c}=\frac{G M}{c^{2}}, \quad \rightarrow \quad \dot{r}_{\max }= \pm \kappa c .
$$

Thus, including the values of the energy conservation constant $\kappa$ into (44) we obtain the following relation:

$$
\dot{r}_{\text {max-hyperbolic }}>\dot{r}_{\text {max-flat }}>\dot{r}_{\text {max-hyperspherical }} \text {. }
$$

Now, including $r_{1}$ and $r_{2}$ from (43) into the accelerating equation of the universe motion (42), we obtain the related accelerations at the radiuses $r_{1}$ and $r_{2}$ :

$$
\begin{aligned}
& \ddot{r}_{1}=-\frac{G M}{r_{1}^{2}}\left(1-\frac{G M}{r_{1} c^{2}}\right)=\frac{\kappa(1+\kappa)^{2} c^{4}}{G M}, \\
& \ddot{r}_{2}=-\frac{G M}{r_{2}^{2}}\left(1-\frac{G M}{r_{2} c^{2}}\right)=\frac{-\kappa(1-\kappa)^{2} c^{4}}{G M} .
\end{aligned}
$$

The first relation in (46) gives the repulsive initial acceleration $\ddot{r}_{1}$ at the radius $r_{1}$ for all scenarios of the universe 
motion. On the other side the acceleration $\ddot{r}_{2}$ at radius $r_{2}$ of the universe motion is attractive for hyperspherical and hyperbolic scenarios of the universe motion, but for flat scenario $(\kappa=1)$ radius $r_{2}$ is going to infinity and the acceleration is going to zero.

From the relations (25) and (43) we can derive the relation between parameters $\kappa$ and $\mathcal{K}$ :

$$
\begin{gathered}
\left(1-\kappa^{2}\right)=\mathcal{K}, \quad \kappa^{2}=1 \rightarrow \mathcal{K}=0 \rightarrow \text { Flat universe }, \\
\kappa^{2}<1 \rightarrow \mathcal{K}>0 \rightarrow \text { Hyperspherical universe, } \\
\kappa^{2}>1 \rightarrow \mathcal{K}<0 \rightarrow \text { Hyperbolic universe. }
\end{gathered}
$$

Here $\kappa$ is the energy conservation constant derived from the Lagrangean (15) and $\mathcal{K}$ is spatial curvature parameter from the Friedmann equations (1).

\section{Limitations of the energy conservation constant $\boldsymbol{\kappa}$.}

For determination of the scenario of the universe motion given by (47), we have to know the limitations of the energy conservation constant $\kappa$. In that sense, one can start with the determination of the radial density $\rho_{\mathrm{r}}$ at the minimal and maximal universe radiuses:

$$
\begin{gathered}
\rho_{r}=\frac{M}{r}, \rho_{r_{\text {max }}}=\frac{M}{r_{\text {min }}}=\frac{(1+\kappa) c^{2}}{G}, \\
\rho_{r_{\text {min }}}=\frac{M}{r_{\text {max }}}=\frac{(1-\kappa) c^{2}}{G}, \\
\frac{\rho_{r_{\text {max }}}}{\rho_{r_{\text {min }}}}=\frac{(1+\kappa)}{(1-\kappa)} \rightarrow \kappa=\frac{\rho_{r_{\text {max }}}-\rho_{r_{\text {min }}}}{\rho_{r_{\text {max }}}+\rho_{r_{\text {min }}}}<1 .
\end{gathered}
$$

From the previous relations we can see the limitations of the energy conservation constant $0<\kappa<1$ and the limitation of the spatial curvature of the space $\mathcal{K}>0$. For that case our universe is a hyperspherical because flat and hyperbolic universes are excluded by limitations of parameters $\kappa$ and $\mathcal{K}$.

\section{CONCLUSION}

New dynamic model of the universe motion, based on the existence of the gravitational energy - momentum tensor (but without cosmological constant 1 ), is presented. As the consequence we obtain the repulsive gravitational force. This repulsive gravitational force could be the source of the dark energy instead of the cosmological constant $\Lambda$. In that sense a dark energy could be seen as a repulsive gravitational energy. The regularity condition of the related line element shows that presented solution has no singularity points. From the universe velocity equation and radial density, $\rho_{r}=M / r$, at the minimal and maximal gravitational radiuses, we obtain the limitations to the energy conservation constant $0<\kappa<1$ and to the spatial curvature of the space $\mathcal{K}>0$. In that case our universe is a hyperspherical because flat and hyperbolic universes are excluded by limitations of parameters $\kappa$ and $\mathcal{K}$. Meanwhile, the presented dynamic model of the universe motion describes the motion after universe creation by Big Bang, at the moment when gravitational repulsive force became dominant in a space - time.
Funding: This work was supported by the National Scientific Foundation of Republic of Croatia (grant number: 120-1201842-3048).

\section{REFERENCES}

[1] A. Friedmann, Über die Krümmung des Raumes, Z.Phys. 10 (1922) 377-386 (in German). A. Friedman, On the curvature of space, General Relativity and Gravitation 31 (1999) 1991-2000.

[2] A. Friedmann, Über die Möglichkeit einer Welt mit konstanter negativer Krümmung des Raumes, Z.Phys. 21 (1924) 326-332 (in German). A. Friedman, On the possibility of a world with constant negative curvature of space, General Relativity and Gravitation 31 (1999) 2001-2008.

[3] G. Lemaître, Expansion of the universe, a homogeneous universe of constant mass and increasing radius accounting for the radial velocity of extra galactic nebulae, Monthly Notices of the Royal Astronomical Society 91 (1921) 483-490.

[4] G. Lemaître, L’Univers en expansion, Annales de la Société Scientifique de Bruxelles A53 (1933) 51-85 (in French).

[5] H.P. Robertson, Kinematics and world structure, Astrophysical Journal 82 (1935) 284-301

[6] H.P. Robertson, Kinematics and world structure II, Astrophysical Journal 83 (1936) 187-201.

[7] H.P. Robertson, Kinematics and world structure III, Astrophysical Journal 83 (1936) 257-271.

[8] A. G. Walker, On Milne's theory of world structure. Proceedings of the London Mathematical Society 242 (1937) 90-127.

[9] L. Bergström, A. Goobar, Cosmology and particle astrophysics, second ed., Sprint, 2006, ISBN 3540329242.

[10] A. Einstein, The Meaning of Relativity, fifth ed., Princ. Univ. Press, Princet., New York, 1955.

[11] Ray d'Inverno, Introducing Einstein's Relativity, Clarendon Press, 1992, ISBN 0198596537.

[12] M. Rees, Just Six Numbers, Orion Book, London, 2000.

[13] M. Lachieze-Rey, J.P. Luminet, Cosmic topology, Physical Reports 254 (1995) 135-214.

[14] G.F.R. Ellis, H. van Elst, Cosmological models, In Marc Lachieze-Rey, Theoretical and Observational Cosmology, NATO Science Series C. 541 (1999) 1-116.

[15] P. Ojeda, H. Rosu, Supersymmetry of FRW barotropic cosmologies, International Journal of Theoretical Physics 45 (2006) 1191-1196.

[16] B, Tersic, Lectures Notes on Astrophysics, Retrieved 20 July (2011) http://nicadd.niu.edu/ btersic/PHYS652_notes.pdf .

[17] Wikipedia, the free encyclopedia, FLRW Metrics, Retrieved 19 March (2012). http://en.wikipedia.org/wiki/Friedmann Lemaitre Robertson Walker Metrics.

[18] Wikipedia, the free encyclopedia. Friedmann Equations, Retrieved 19 March (2012). http://en.wikipedia.org/wiki/Friedmann_equations .

[19] A.H. Guth, Inflationary universe: A possible solution to the horizon and flatness problems, Phys. Rev. D23 (1981) 347; New York Academy of Sciences Magazine, Jan./Febr., (2002).

[20] S. Perlmutter et al., Nature 391 (1998) 51; Riess A. et al., Astron. J. 116 (1998) 1009

[21] R.R. Caldwell et al., Phys. Rev. Lett. 80 (1998) 1582, Caldwell R. R., astro-ph/9908168.

[22] L. Parker, A. Raval, Phys. Rev. D 60 (1999) 063512; 60 (1999) 123502; 62 (2000) 083503, Retrieved 19 March (2012).

[23] P.J. Steinhard, N. Turok, http://feynman.princeton.edu/ steinh/, hepth/ 0111030, Science (2002), hepth/0111098, Phys. Rev. D (2002).

[24] B.M. Novakovic, D.B. Novakovic, A.B. Novakovic, Cosmological constant problem solution valid for both Planck's and cosmological scales. AIP Conference Proceedings 839

(2006) 144 https://doi.org/10.1063/1.2216624

[25] B. Sterling, Surprise! Our little corner of the universe is even smaller than we thought (2003). www.wired.com/wired/archive/11.11/view.html?pg=4.

[26] G.M. Kremer, Cosmological models described by a mixture of der Waals fluid and Dark energy, Phys. Rev. D 68 (2003) 123507.

[27] S.E. Jorás, T.J. Stuchi, Chaos in a closed Friedmann Robertson Walker universe: An imaginary approach, Phys. Rev. D 68 (2003) 123525.

[28] M. Susperregi, Dark energy and Dark matter from an inhomogeneous dilaton, Phys. Rev. D 68 (2003) 123509. 
Energy Momentum Tensor generates Repulsive Gravitational Force

[29] P.G. Castro, M. Douspis, P.G. Ferreira, Scale of homogeneity of the universe from WMAP, Phys. Rev. D 68 (2003) 127301.

[30] P.P. Avelino, C. J.A.P. Martins, C. Santos, E.P.S. Shellard, Topological defects: A problem for cyclic universe, Phys. Rev. D 68 (2003) 123502.

[31] J. Khoury, P.J. Steinhardt, N. Turok, Designing cyclic universe models, Phys. Rev. Lett. 92 (2004) 031302.

[32] J. E. Lidsey, Physical Review D 70 (2004) 041302.

[33] T. Clifton, C. Clarkson, P. Bull, Isotropic blackbody: Cosmic microwave background radiation as evidence for a homogeneous universe, Phys. Rev. Lett. 109 (2012) 051303.

[34] A. Pisani, et al., Counting voids to probe dark energy, Phys. Rev. D 92 (1015) 083531 .

[35] M. Lincoln, A. Wasser, Spontaneous creation of the Universe Ex Nihilo, Physics of the Dark Universe 2 (2013) 195-199. https://doi.org/10.1016/j.dark.2013.11.004

[36] M. Baldi, Dark Energy simulations, Dark Universe 1 (2012) 162-193. https://doi.org/10.1016/j.dark.2012.10.004

[37] M. Kuhlen, M. Vogelsberger, R. Angulo, Numerical simulations of the dark universe: State of the art and the next decade, Dark Universe 1 (2012) 50-93. https://doi.org/10.1016/j.dark.2012.10.002

[38] P. Huang, F. F. Yuan, Uniformly expanding vacuum: A possible interpretation of the dark energy, Physics of the Dark Universe 12 (2016) 45-49

[39] S. GHAFFARI, H. MORADPOUR, VALDIR B. BEZERRA, J.P. MORAISGRAÇA, I.P. LOBO, TSALLIS HOLOGRAPHIC DARK ENERGY IN THE BRANE COSMOLOGY. HTTPS://DOI.ORG/10.1016/J.DARK.2018.11.007

[40] R. von Marttens, L. Casarini, D.F. Mota, W. Zimdahl, Cosmological constraints on parametrized interacting dark energy. https://doi.org/10.1016/j.dark.2018.10.007

[41] A. Casalino, M. Rinaldi, L. Sebastiani, S. Vagnozzi, Mimicking dark matter and dark energy in a mimetic model compatible with GW170817, Physics of the Dark Universe 22 (2018) 108-115. https://doi.org/10.1016/j.dark.2018.10.001

[42] J. Baez, The Planck length (1999). (http://math.ucr.edu/home/baez/planck/node2.html)

[43] CODATA: Constants, Planck length, US National Institute of Standard and Technology, June (2015). (http://physics.nist.gov/cgi-bin/cuu/Value?plkl)

[44] R. Bowley, L., Eaves, Planck Length (2010). (http://www.sixtysymbols.com/videos/plancklength.htm)

[45] CODATA Value, Planck mass, US National Institute of Standard and Technology, June (2015). (http://physics.nist.gov/cgi-bin/cuu/Value?plkm)

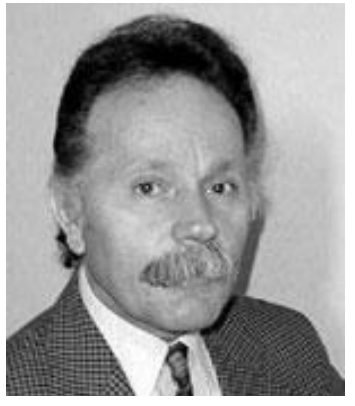

Branko Novakovic is a Professor emeritus at FSB - University of Zagreb, Croatia. Prof. Novakovic received his $\mathrm{PhD}$ from the University of Zagreb in 1978. His research of interest includes physics, control systems, robotics, neural networks, and fuzzy control. He is author of three books; Relativistic Alpha Field Theory (RAFT, e-book, 2016), Control Methods in Robotics, Flexible Manufacturing Systems and Processes (1990), Control Systems (1985) and the first co-author of a book Artificial Neural Networks (1998). He has published over 235 research papers in his research of interest. 